\title{
Improving Efficiency While Improving Patient Care in a Student-Run Free Clinic
}

\author{
Jason S. Lee, ${ }^{1}$ BS, Kristen Combs, ${ }^{1}$ BS, KNIGHTS Research Group 2016, and \\ Magdalena Pasarica, $M D, P h D$
}

Introduction: Student-run free clinics (SRFCs) have the capacity to decrease health care inequity in underserved populations. These facilities can benefit from improved patient experience and outcomes. We implemented a series of quality improvement interventions with the objectives to decrease patient wait times and to increase the variety of services provided.

Methods: A needs assessment was performed. Problems related to time management, communication between staff and providers, clinic resources, and methods for assessing clinic performance were identified as targets to reduce wait times and improve the variety of services provided. Seventeen interventions were designed and implemented over a 2 -month period.

Results: The interventions resulted in improved efficiency for clinic operations and reduced patient wait times. The number of specialty providers, patient visits for specialty care, lifestyle education visits for disease prevention and treatment, free medications, and free laboratory investigations increased to achieve the goal of improving the availability and the variety of services provided.

Conclusions: We demonstrated that it is feasible to implement successful quality improvement interventions in SRFCs to decrease patient wait times and to increase the variety of services provided. We believe that the changes we implemented can serve as a model for other SRFCs to improve their performance. (J Am Board Fam Med 2017;30:513-519.)

Keywords: Access to Health Care; Health Care Disparities; Continuity of Care; Education; Patient; Health Resources; Medical Students; Needs Assessment; Quality Improvement; Quality of Health Care; Student Run Clinic; Vulnerable Populations

Student-run free clinics (SRFCs) are institutions operated primarily by medical students, with oversight by attending licensed physicians, that provide health care services to poor or uninsured patients. These clinics, as well as standard free clinics, are essential to communities with low levels of health care access and few resources because they help reduce health care inequity. ${ }^{1-4}$ The rapid increase in homelessness over the past 20 years, the shortage

This article was externally peer reviewed.

Submitted 14 February 2017; revised 14 February 2017; accepted 13 March 2017.

From the Department of Medical Education, University of Central Florida College of Medicine, Orlando.

Funding: none.

Conflict of interest: none declared.

Corresponding author: Jason S. Lee, BS, Department of Medical Education, University of Central Florida College of Medicine, 6850 Lake Nona Blvd, Orlando, FL 32827 (Email: jasonsl@knights.ucf.edu).

${ }^{1} \mathrm{JSL}$ and $\mathrm{KC}$ contributed equally to this work. of health care professionals in underserved areas, and the difficulty some individuals have in obtaining affordable health care insurance are just a few of the many reasons that free clinics are indispensable to society. ${ }^{5,6}$ It is well known that a lack of access to health care is associated with poor health outcomes. $^{1,4}$

SRFCs should continuously seek to improve patient outcomes. ${ }^{7}$ Such clinics are increasingly being used as health care access points for the underserved, as well as avenues through which medical students and resident physicians can enhance their education. ${ }^{8-11}$ Several recent studies analyzed the level of care, patient satisfaction, and services offered at various SRFCs to improve patient care and student education. The Weill Cornell Community Clinic was 1 of the first SRFCs to incorporate the patient-centered medical home model, which emphasizes longitudinal experience in continuity of care, work in interdisciplinary teams, practice with 
a variety of encounters, and exposure to medical informatics. Use of this model resulted in a high level of student satisfaction with the clinical experience. ${ }^{12}$ Another SRFC at the Pritzker School of Medicine determined that the presence of fourthyear medical students improves both student comfort with physicians and overall satisfaction. ${ }^{13}$ Butala et $\mathrm{al}^{14}$ described several interventions used at HAVEN Free Clinic to improve the quality of preventive care, including the implementation of a medical record specialist position. The Shade Tree Family Clinic, an SRFC at Vanderbilt University, implemented a Web-based pharmaceutical tracking system to improve management of its medication formulary. ${ }^{7}$

The SFRC at University of Central Florida College of Medicine (the KNIGHTS Clinic [Keeping Neighbors in Good Health Through Service]) is funded by the Diebel Legacy Fund at Central Florida Foundation, a nonprofit organization. The SRFC is coordinated and staffed by medical students at the University of Central Florida College of Medicine under the supervision of volunteer physicians and pharmacists. Underserved patients in Orlando receive free health care services, health promotion, disease prevention, social support, case management, and appropriate referrals to medical and social resources in the community. The SRFC provides continuity of care and interdisciplinary care by medical providers, pharmacy personnel, and social workers. Furthermore, the SRFC provides primary care and specialty care. It also provides opportunities for students to become involved in patient care, medical record management, phlebotomy, and numerous other procedures.

Specialty board positions in various fields include student-directors, student-providers (second-year medical students), supervising student-providers (third- or fourth-year medical students), student-educators, student-pharmacists, student-informaticists, student-recruiters, student-phlebotomists, and student-researchers. Student-providers and supervising student-providers offer care to patients under the close supervision of volunteer attending physicians. Studenteducators provide education on health issues for disease prevention and treatment. Student-pharmacists provide sample medications and use the Prescription Assistance Program (PAP). Studentphlebotomists draw blood, under professional supervision, if laboratory testing is required. Stu- dent-informaticists assist student-providers and physicians with the electronic medical record (EMR). Student-recruiters find volunteer attending physicians in the community and assign them to specific clinic dates. Student-schedulers assign student-providers and supervising student-providers to each clinic.

SRFCs, like other health care institutions, have methodologies that should be continuously assessed in order to analyze their efficacy. The aspect of SRFCs most commonly viewed as needing improvement is patient wait times. ${ }^{12,15,16}$ To our knowledge, no study to date has thoroughly described clinic operation methods that may benefit patient wait time and clinic efficiency. Our SRFC is committed to providing optimal patient-centered medical care and improving the education of our medical students and physician volunteers.

We performed a needs assessment to identify problems related to time management, communication between staff and providers, clinic resources, and methods for assessing clinic performance. Specifically, we identified that (1) we roomed patients late after they arrived; (2) splitting the studentproviders' responsibilities across 9 teams did not foster optimal communication and recognition of duties; (3) patients spent excess time waiting for the overbooked attending physician to discuss his or her management and discharge plan; (4) studentproviders spent excess time processing their duties because of a lack of readily available resources; and (5) baseline data were needed to improve the quality and efficiency of patient care in the clinic. We aimed to decrease patient wait times and increase the variety of services provided at SRFCs by implementing a series of quality improvement interventions.

\section{Methods}

\section{Problems and Quality Improvement Interventions}

In February 2015, overall clinic performance was analyzed and a needs assessment was performed. Several interventions to address each problem were identified (Table 1). These interventions were implemented over the next 2 months. The analysis compared outcomes from March 2014 to February 2015 with data from March 2015 to February 2016. If data from 2014 to 2015 were not collected, then only 2015 to 2016 data were analyzed and presented. Means, standard deviations, and when ap- 
Problems with time management and communication Patients were roomed late after arrival.

Student-providers' responsibilities were split into 9 teams without proper communication and recognition of duties, which increased patient wait time.

Patients were waiting for the overbooked attending physician to discuss the patient's medical management and discharge plan.

Problems with clinic resources

Students providing care were slow in processing their duties due to the lack of readily available information.

Problems with assessing clinic performance

No baseline data were available for use to improve the quality and efficiency of patient care in the clinic.
All students were instructed to arrive at the clinic at least 30 minutes before the clinic start time in order to review the patient's chart. Second-year students were instructed to review the patient's chart a day in advance of the patient's visit.

A board member was designated to notify the student-providers of their patient's arrival in order to decrease the time spent in the waiting room.

Each team was instructed to record the time in and out of the patient room.

Student-care team members adopted the role of measuring vital signs (from student-providers) in order to decrease the wait time between vital signs measurement and the start of history taking.

Student-providers were instructed to provide student-educators with an "education form" to communicate patient education needs more effectively.

Student-providers were instructed to provide student-pharmacists with a "medication form" to communicate needed medications more effectively.

The number of patients seen by each physician was decreased from 3 or 4 to 2 or 3 patients.

The number of physicians participating at each clinic was increased to improve physician availability for each patient.

Physicians' schedules were confirmed at least 2 months before their clinic date to decrease the number of physician cancellations.

The pool of supervising student-providers was increased by integrating the internal/family medicine third-year clerkship students into the clinic, thereby increasing the number of patients seen per clinic.

Periodic surveys were sent to third- and fourth-year students to determine their availability and decrease cancellation frequency.

A single physician faculty advisor was designated to monitor and manage laboratory results, alongside the student-providers responsible for each patient, to decrease the workload outside of clinic for the other volunteer attending physicians.

Student-educators were trained on lifestyle education using a free interactive website.

A written guide for applying to Prescription Assistance Program (PAP) was developed to decrease the wait time between prescribing the medicine and completing the form.

Student-pharmacists were instructed to verify the PAP medications at the beginning of each clinic in order to decrease wait time for medications dispensed by the PAP.

An EMR user guide was created and distributed to student-providers (as a self-learning module and printed resource) in order to decrease time spent documenting in the patient chart.

The number of student-informaticists was increased from 2 to 4 per clinic in order to decrease documentation time.

A research committee was created and met periodically with the purpose of designing research studies to evaluate patient satisfaction and educational benefits for the volunteers.

All clinic board members were instructed to collect, track, and analyze their own data (time of appointments, number of patients, patients receiving education, physician-to-patient ratios, etc.)

In February 2015, overall clinic performance was analyzed and a needs assessment was performed. These interventions were implemented over the next 2 months. The analysis compared outcomes from March 2014 to February 2015 with data from March 2015 to February 2016.

EMR, electronic medical record; PAP, Prescription Assistance Program. 
Figure 1. Change in the recruitment of volunteer physicians and supervising student-providers from 2014 to 2015 to 2015 to 2016. A: The change in primary and specialty care physician recruitment. B: The number of upper-level (third- and fourth-year) medical students volunteering as supervising student-providers. Values are represented as absolute numbers.
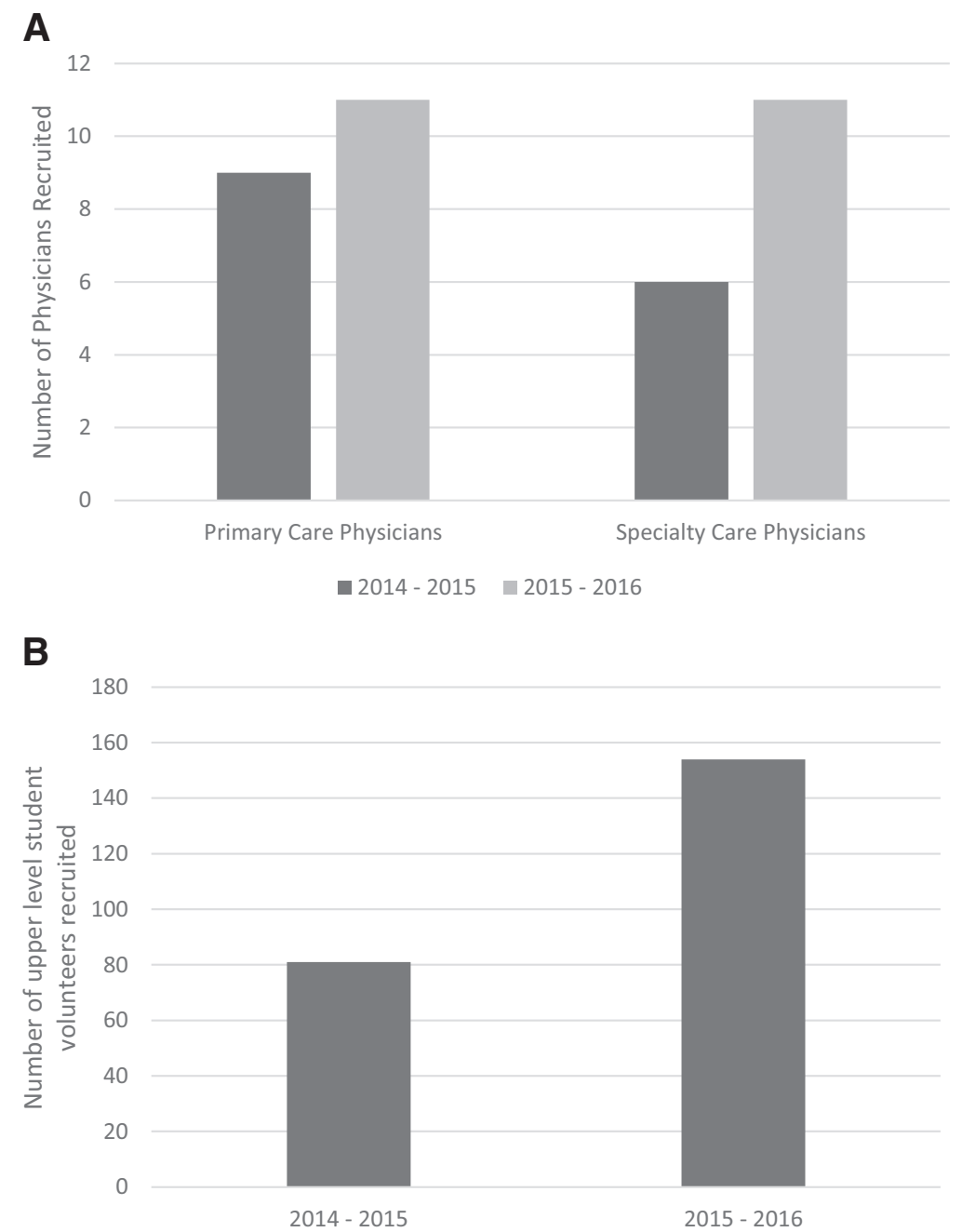

plicable, percentages were calculated. When applicable, a $t$ test was performed to compare the results before and after the interventions. We considered $P<.05$ to indicate statistical significance.

\section{Results}

\section{Change in the Availability and Variety of Services} Provided from 2014 to 2015 to 2015 to 2016

To improve the availability of medical appointments, we implemented interventions designed to increase the providers recruited (Table 1). The total number of newly recruited volunteer physicians increased from 15 to 22 (Figure 1A), as did the number of medical students recruited as supervising student-providers (Figure 1B). These im- provements resulted in an increase in the number of patient appointments offered per clinic (from $4 \pm 1$ to $6 \pm 2 ; P<.05)$ and patient appointments per year (from 101 to 114).

To improve the variety of services provided, we implemented interventions designed to increase the number of specialty providers, patient visits for specialty care, lifestyle education visits for disease prevention and treatment, free medications, and free laboratory investigations (Table 1). The number of specialty volunteer physicians newly recruited to provide a variety of specialty patient care increased from 6 to 10, which was accompanied by an increase in the proportion of specialty care visits (from $18 \%$ to $24 \%$ ). During this period, 54 patients 
Figure 2. Medical appointment duration in 2014 to 2015 versus 2015 to 2016. "Patient-provider visit contact time" represents the amount of time patients spent with the providers. "Patient wait time" represents the time spent by the patient in the waiting room and clinic room waiting for providers, procedures, education, lab tests, or medications. "Patient total visit time" represents the time between check-in and check-out. Values are presented as mean \pm standard deviation. $* P<.05$.

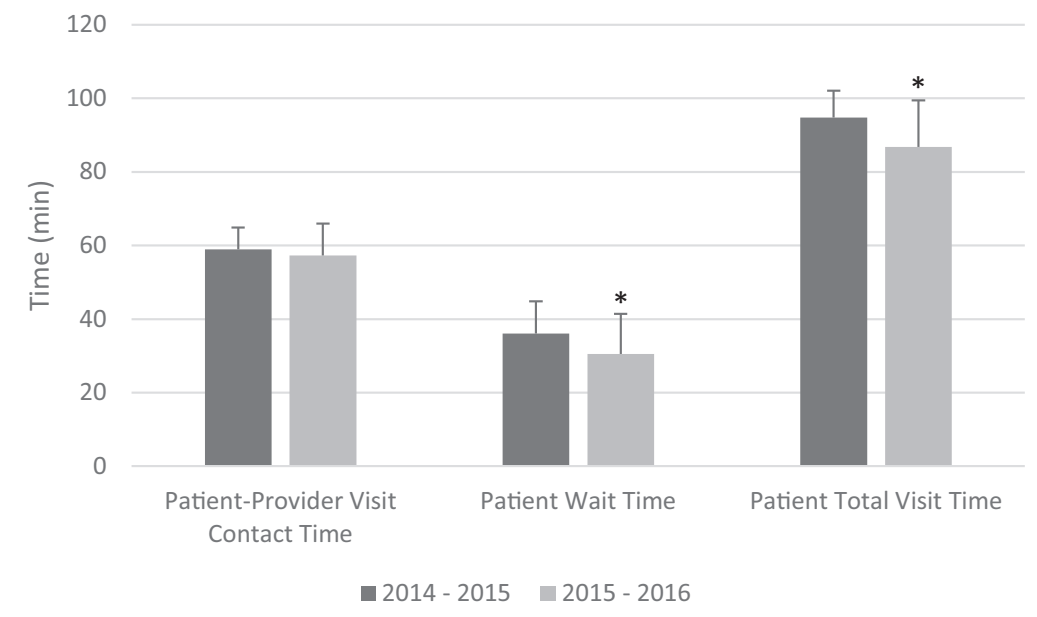

received lifestyle education for disease prevention and treatment. This lifestyle education covered a total of 10 topics (with an average of 4 topics/ patient counseled). A total of 30 patients received free medications and 16 patients received free laboratory investigations.

\section{Change in Clinic Efficiency and Patient Wait Times from 2014 to 2015 to 2015 to 2016}

We defined patient wait time as the time spent by a patient in the waiting room and clinic room waiting for providers, procedures, education, lab tests, and medications. To decrease patient wait times, we implemented several interventions simultaneously to improve clinic efficiency (Table 1).

To eliminate the need for patients to spend unnecessary time waiting for orders to be clarified, we successfully implemented several interventions (Table 1) to improve communication between the teams. Considering the total number of medication orders, the use of a newly developed written order form for dispensing sample medications increased from $25 \%$ to $100 \%$.

We implemented several educational interventions to improve the knowledge of students providing patient education (Table 1). We also implemented educational interventions to assist student in completing prescription assistance programs applications and documenting patient visit information in the EMR (Table 1). These interventions helped decrease the patient wait time during the completion of these tasks. In addition, we developed a quality improvement research committee to design tools for measuring the efficacy of the interventions implemented.

Patient wait times as well as total patient visit length decreased significantly after the implementation of these interventions $(P<.05)$. Importantly, the amount of patient-provider contact during a visit remained stable (data were not significant) (Figure 2).

\section{Discussion}

We designed and implemented 17 quality improvement interventions at the KNIGHTS Clinic. These interventions resulted in improved efficiency for clinic operations and a decrease in patient wait times. The number of specialty providers, patient visits for specialty care, lifestyle education visits for disease prevention and treatment, free medications, and free laboratory investigations increased to achieve the aim of improving the availability and variety of services provided.

The first problem addressed was that patients were waiting to be roomed after they were checked in. This is a common problem in most clinics all over the United States. We addressed this issue by requiring all students arrive to clinic at least 30 minutes before the first visit time in order to review 
the first patient's chart. We also instructed studentproviders to review a patient's chart a day in advance of his or her visit. Students were thus more familiar with his or her patient's medical issues before the patient arrived. As a result, we roomed patients almost immediately after checking in. Because of the easy application of this change in our clinic, we believe that this change can be implemented at other SRFCs, as well as traditional private or daytime free outpatient clinics.

Deficient communication and recognition of specific duties is a problem that commonly occurs in clinics. Recording time in and time out of patient rooms, engaging in discussion of duties and specific appointment durations, and implementing written transfers of care between teams contributed to more efficient use of patient-provider time, as evidenced by the decreased total patient visit times.

Overbooking supervising providers is a common problem occurring not only in SRFCs but also in traditional clinics. By optimizing providers' time in the clinic, improving communication regarding visit scheduling, and increasing opportunities for providers, we successfully recruited more physicians and supervising-student providers. Even if SRFCs are run by students, the presence of attending physicians at SRFCs is essential to clinic operations, as medical care can only be provided under the supervision of a licensed physician. Respecting the valuable time of volunteer physicians is important. We improved physician retention and satisfaction by clearly communicating clinic dates, scheduling reminders, and efficiently using volunteer physicians' time in the clinic. We increased the recruitment of specialty care providers, which increased the diversity of medical conditions that can be addressed at our clinic. We found that capping the number of patients seen by each physician per clinic assignment allowed attending physicians to spend more time with each of their patients and reduced patient wait times. We realize that this restriction may not be a viable option for traditional private clinics, but it certainly constitutes an option for SRFCs and perhaps other free clinics.

We addressed another common problem in our clinic: the lack of readily available information for the student-educators. Using information provided on the National Institutes of Health's Nutrition in Medicine website, ${ }^{17}$ we better educated studenteducators and our patients about the nutritional management of diseases using a national-level ap- proach. By providing patients with sunscreen and sun-safety education, we addressed a health care concern that is particularly relevant to this vulnerable population. Sunscreen use and sun-safety education is often ignored in busy general medical practices. ${ }^{18} \mathrm{We}$ gave our patients the opportunity to take precautions aimed at preventing diseases by encouraging multivitamin use to meet daily nutritional requirements and by advocating for vaccine administration. We further applied specific, measurable, attainable, realistic, and time-sensitive goal setting for patients, which fostered more effective evidence-based patient counseling. ${ }^{19}$

The user guide for charting in the EMR and the presence of more student-informaticists on site increased the accuracy and efficiency of medical charting. An EMR user guide is usually available in clinics, especially after a new EMR system is implemented. This intervention improved student satisfaction relating to his or her volunteer experience and can be readily applied to other traditional clinics facing similar charting problems with their EMRs.

Finally, we are aware that one cannot improve clinic performance if no baseline data are available to clarify what the problems are and how they are resolved. We created a research committee to help design and implement studies with the purpose of improving the quality of medical care, the efficiency of clinic operations, and patient satisfaction at our clinic. Our board members presented at the National Conference of Student Run Free Clinics 8 posters on our clinic improvements. This intervention would be helpful for both SRFCs and traditional clinics.

Our study has several limitations. We did not compare patient satisfaction before and after the interventions because of lack of data for the year 2014 to 2015 . However, we did receive verbal comments from patients reporting improved satisfaction with clinic experience in the 2015 to 2016 academic year. Because we deployed all interventions within a 2 -month period, we cannot determine which intervention was most effective; however, this limitation is outweighed by the benefit of improved clinic performance in a timely manner.

\section{Conclusions}

The interventions implemented in our SRFC resulted in improved clinic efficiency, decreased pa- 
tient wait times, increased availability of services, and increased variety of services provided. While problems with communication and time management, clinic resources, and performance assessment are common within SRFCs, they are also encountered within other traditional private or free outpatient clinics. Therefore, we suggest that the interventions used by our SRFC be used by other SRFCs and traditional clinics to improve clinic efficiency and patient care.

The authors thank the Diebel Legacy Fund at Central Florida Foundation, Grace Medical Home, and the University of Central Florida College of Medicine for their continuous support.

KNIGHTS Research Group 2016: Sean All, BS, Christopher Atkins, MS, Kathyrn Baker, BS, MS, Kyle Cox, BS, Anand Desai, BS, Madeline Goldberg, MS, Karen Lu, BA, Jheel Pandya, BS, Priya Patel, BS, Priyal Patel, BA, Michael R Povlow BS, Stephen Rineer, BS, Michael Simpson, BS, Bryan Thiel, BS, Judith Simms-Cendan, MD, PhD, University of Central Florida College of Medicine, 6850 Lake Nona Blvd, Orlando, FL 32827, Medical Education.

To see this article online, please go to: http://jabfm.org/content/ 30/4/513.full.

\section{References}

1. Andrulis DP. Access to care is the centerpiece in the elimination of socioeconomic disparities in health. Ann Intern Med 1998;129:412-6.

2. Cooper LA, Hill MN, Powe NR. Designing and evaluating interventions to eliminate racial and ethnic disparities in health care. J Gen Intern Med 2002;17:477-86.

3. Liu JJ. Health professional shortage and health status and health care access. J Health Care Poor Underserved 2007;18:590-8.

4. Oliver A, Mossialos E. Equity of access to health care: outlining the foundations for action. J Epidemiol Community Health 2004;58:655-8.

5. Moskowitz D, Glasco J, Johnson B, Wang G. Students in the community: an interprofessional student-run free clinic. J Interprof Care 2006;20:254-9.

6. Niescierenko ML, Cadzow RB, Fox CH. Insuring the uninsured: a student-run initiative to improve access to care in an urban community. J Natl Med Assoc 2006;98:906-11.

7. Rosenbaum BP, Patel SG, Guyer DL, et al. The pharmaceutical management system at Shade Tree
Family Clinic: a medical student-run free clinic's experience. Inform Health Social Care 2008;33: 151-7.

8. Batra P, Chertok JS, Fisher CE, Manseau MW, Manuelli VN, Spears J. The Columbia-Harlem Homeless Medical Partnership: a new model for learning in the service of those in medical need. J Urban Health 2009;86:781-90.

9. Meah YS, Smith EL, Thomas DC. Student-run health clinic: novel arena to educate medical students on systems-based practice. Mt Sinai J Med 2009;76: 344-56.

10. Simpson SA, Long JA. Medical student-run health clinics: important contributors to patient care and medical education. J Gen Intern Med 2007;22: 352-6.

11. Swartz MK. The contributions of student-run free clinics. J Pediatr Health Care 2012;26:397.

12. Riddle MC, Lin J, Steinman JB, et al. Incorporating the principles of the patient-centered medical home into a student-run free clinic. Adv Med Educ Pract 2014;5:289-97.

13. Choudhury N, Khanwalkar A, Kraninger J, Vohra A, Jones K, Reddy S. Peer mentorship in student-run free clinics: the impact on preclinical education. Fam Med 2014;46:204-8.

14. Butala NM, Chang H, Horwitz LI, Bartlett M, Ellis P. Improving quality of preventive care at a studentrun free clinic. PLoS One 2013;8:e81441.

15. Clark JS, Bollaert A, Sills SO, Clark JH, Norris D. Patient perception of care received by students at the Jackson Free Clinic. J Miss State Med Assoc 2014; 55:113-8.

16. Ellett JD, Campbell JA, Gonsalves WC. Patient satisfaction in a student-run free medical clinic. Fam Med 2010;42:16-18.

17. Nutrition in Medicine. Evidence-based clinical nutrition education for medical students, residents, fellows and other physicians [homepage]. Available from: http://nutritioninmedicine.org/. Accessed February 7, 2017.

18. Bandi P, Cokkinides VE, Weinstock MA, Ward EM. Physician sun protection counseling: prevalence, correlates, and association with sun protection practices among US adolescents and their parents, 2004. Prev Med 2010;51:172-7.

19. Revello K, Fields $W$. An educational intervention to increase nurse adherence in eliciting patient daily goals. Rehabil Nurs 2015;40:320-6. 